\title{
Superresolution imaging: a survey of current techniques
}

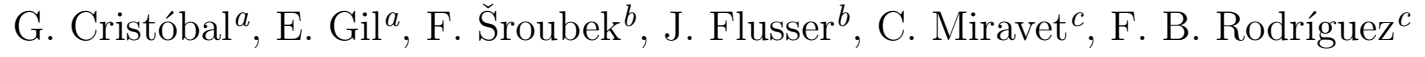 \\ ${ }^{a}$ Instituto de Optica, Serrano 121, 28006 Madrid, Spain; \\ ${ }^{b}$ Institute of Information Theory and Automation of the ASCR, Pod vodárenskou věží 4 \\ Prague, Czech Republic; \\ ${ }^{c}$ Escuela Politécnica Superior, Universidad Autónoma, Madrid, Spain
}

\begin{abstract}
Imaging plays a key role in many diverse areas of application, such as astronomy, remote sensing, microscopy, and tomography. Owing to imperfections of measuring devices (e.g., optical degradations, limited size of sensors) and instability of the observed scene (e.g., object motion, media turbulence), acquired images can be indistinct, noisy, and may exhibit insufficient spatial and temporal resolution. In particular, several external effects blur images. Techniques for recovering the original image include blind deconvolution (to remove blur) and superresolution (SR). The stability of these methods depends on having more than one image of the same frame. Differences between images are necessary to provide new information, but they can be almost unperceivable. State-of-the-art SR techniques achieve remarkable results in resolution enhancement by estimating the subpixel shifts between images, but they lack any apparatus for calculating the blurs. In this paper, after introducing a review of current SR techniques we describe two recently developed SR methods by the authors. First, we introduce a variational method that minimizes a regularized energy function with respect to the high resolution image and blurs. In this way we establish a unifying way to simultaneously estimate the blurs and the high resolution image. By estimating blurs we automatically estimate shifts with subpixel accuracy, which is inherent for good SR performance. Second, an innovative learning-based algorithm using a neural architecture for SR is described. Comparative experiments on real data illustrate the robustness and utilization of both methods.
\end{abstract}

Keywords: superresolution, deconvolution, energy minimization, probabilistic neural network, sequence processing.

\section{INTRODUCTION}

Imaging devices have limited achievable resolution due to many theoretical and practical restrictions. An original scene with a continuous intensity function $o(x, y)$ warps at the camera lens because of the scene motion and/or change of the camera position. In addition, several external effects blur images: atmospheric turbulence, camera lens, relative camera-scene motion, etc. We will call these effects volatile blurs to emphasize their unpredictable and transitory behavior, yet we will assume that we can model them as convolution with an unknown point spread function (PSF) $h(x, y)$. This is a reasonable assumption if the original scene is flat and perpendicular to the optical axis. Finally, the CCD discretizes the images and produces digitized noisy image $g(i, j)$, which we refer to as a low-resolution (LR) image, since the spatial resolution is too low to capture all the details of the original scene. For one single observation $g(i, j)$ the problem is heavily underdetermined and lacks stable solution. To partially overcome the equivocation of the problem, we can take $K(K>1)$ images of the original scene and face the so-called multichannel (multiframe) problem. The acquisition model then becomes

$$
g_{k}(i, j)=D\left(\left[h_{k} * W_{k}(o)\right](x, y)\right)+n_{k}(i, j),
$$

where $k=1, K$ is the acquisition index, $n_{k}(i, j)$ is additive noise and $W_{k}$ denotes the geometric deformation (warping), in general different for each acquisition. $D(\cdot)$ is the decimation operator that models the function of CCD sensors. It consists of convolution with a sensor PSF followed by a sampling operator, which we define as multiplication by a sum of delta functions placed on a grid. The above model is the state of the art as it takes all possible degradations into account.

Further author information: (Send correspondence to G.C.)

G.C.: E-mail: gabriel@optica.csic.es, Telephone: 34915616800

Advanced Signal Processing Algorithms, Architectures, and Implementations XVIII,

edited by Franklin T. Luk, Proc. of SPIE Vol. 7074, 70740C, (2008) · 0277-786X/08/\$18 · doi: 10.1117/12.797302

Proc. of SPIE Vol. 7074 70740C-1 


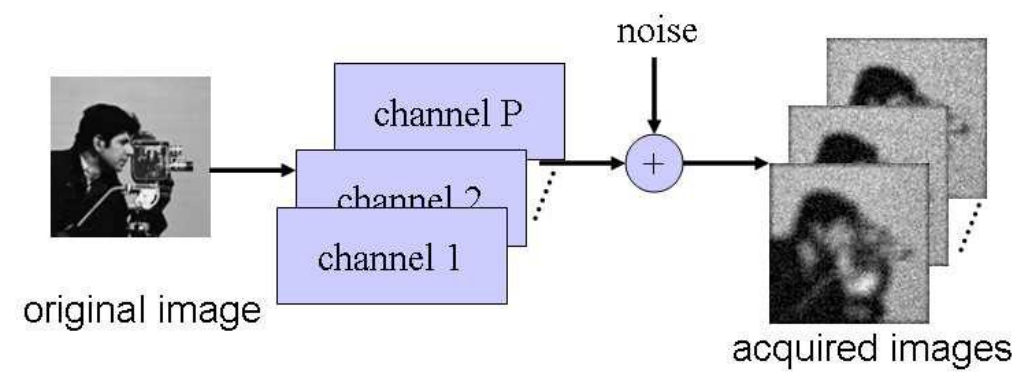

Figure 1: Image acquisition model

Superresolution (SR) is the process of combining a sequence of LR images in order to produce an image or sequence of higher resolution. From the earliest algorithm proposed by Tsai and Huang, ${ }^{12}$ super-resolution has attracted a growing interest as a purely computational means to increase imaging sensors performance. It is unrealistic to assume that the superresolved image can recover the original scene $o(x, y)$ exactly. A reasonable goal of SR is a discrete version of $o(x, y)$, which has higher spatial resolution than the resolution of the LR images and which is free of the volatile blurs (deconvolved). In the paper, we will refer to this superresolved image as a high resolution (HR) image $f(i, j)$ and the ratio between the size of the sought HR image and input LR image will be called a $S R$ factor. The standard SR approach consists of subpixel registration, overlaying the LR images on an HR grid, and interpolating the missing values. The subpixel shift between images thus constitutes an essential feature. We will demonstrate that considering volatile blurs in the model explicitly brings about a more general and robust technique, with the subpixel shift being a special case thereof.

The acquisition model in Eq. 1 embraces three distinct cases frequently encountered in literature. First, removal of the geometric degradation $W_{k}$ is a registration problem. Second, if the decimation operator $D$ and the geometric transform $W_{k}$ are not considered, we face a multichannel (or multiframe) blind deconvolution (MBD) problem. Third, if the volatile blur $v_{k}$ is not considered or assumed known, and $W_{k}$ is suppressed except to subpixel translations, we obtain a classical SR formulation. In practice, it is crucial to consider all three cases at once. This is described in the fist part of this paper under the term blind superresolution (BSR).

Research on intrinsically MBD methods has begun fairly recently; refer to ${ }^{2-6}$ for a survey and other references. The MBD methods can directly recover the blurring functions from the degraded images alone. We further developed the MBD theory in ${ }^{7}$ by proposing a blind deconvolution method for images, which might be mutually shifted by unknown vectors. To make this brief survey complete, we should not forget to mention a very challenging problem of shift-variant blind deconvolution, that was considered in..$^{8,9}$

A countless number of papers address the standard SR problem. A good survey can be found for example in. ${ }^{13,54}$ Most super-resolution methods ${ }^{14,54}$ fall into one of the following four categories: frequency domain, projection onto convex set (POCS), Maximum a Posteriori (MAP) and two-step, scattered interpolation followed by deblurring, methods. Frequency domain methods ${ }^{11,12}$ were historically the first developed. They are based on the shifting property of the Fourier transform and the assumption that the original high-resolution image is band-limited. Their application is largely restricted to cases where only translational motion is present, limiting considerably their use in many practical scenarios. From the plethora of SR algorithms proposed we focused this survey study in two alternative approaches that have been analyzed in depth by the authors: 1) BSR method and 2) Neural-network based method.

First, let review some background about the BSR method. Current MBD techniques require no or very little prior information about the blurs, they are sufficiently robust to noise and provide satisfying results in most real applications. However, they can hardly cope with the decimation operator, which violates the standard convolution model. On the contrary, state-of-the-art SR techniques achieve remarkable results of resolution enhancement in the case of no blur. They accurately estimate the subpixel shift between images but lack any apparatus for calculating the blurs. Recently in ${ }^{30}$ we proposed a unifying method that simultaneously estimates the volatile blurs and HR image. The only prior knowledge required are estimates of the blur size and level of noise in the LR images, which renders it a truly BSR method. The key idea was to determine subpixel shifts 
by calculating volatile blurs. As the volatile blurs are estimated in the HR scale, positions of their centroids correspond to sub-pixel shifts. Therefore by estimating blurs we automatically estimate shifts with sub-pixel accuracy, which is essential for good performance of SR. We showed that the blurs in the HR scale can be recovered from the LR images up to small ambiguity, which is a generalization of results obtained for blur estimation in the MBD case and which we have proposed earlier in. ${ }^{25,30}$ This complex SR problem was solved by minimizing a regularized energy function, where the regularization was carried out in both the image and blur domains. The image regularization is based on variational integrals, and a consequent anisotropic diffusion with good edge-preserving capabilities. The blur regularization term is based on our generalized result of blur estimation in the SR case. To tackle the minimization task, we used an alternating minimization approach consisting of two simple linear equations.

The second surveyed method in this work is a two-step learning-based super-resolution technique. ${ }^{38}$ The algorithm is based on the application of scattered-point interpolation on projected sequence data, followed by a filtering operation to restore degradations associated to sequence pixel size and residual errors introduced by interpolation. The scattered-point interpolation module has been implemented using a novel hybrid neural network architecture, enabling the processing of synthetic sequences to learn optimum distance-based interpolation functions for different noise levels in the input sequence. The restoration process is carried out by application of an optimum linear filter operation to the scattered-point interpolated image. The filter coefficients, which are assumed to be rotationally symmetric, have been determined by minimizing on synthetic data the squared differences between high-resolution images and restoration of first-step interpolated images. This introduced an effective coupling between both steps of the super-resolution method, the lack of which is a common criticism of two-step methods. Experimental results showed significant improvements, both in visual and RMS error terms, over Wiener filter restorations adapted only to correct lens and detector degradations. As in two-step methods in general, this super-resolution scheme implicitly assumes that all sequence frames are affected by the same space-invariant degradation, which is caused exclusively by low-resolution optics and detector blurs, and have a similar noise level. Being targeted to achieve near real-time superresolution execution on input imagery of good quality, in particular no provisions are made to try to identify and restore the effect of common degradations such as those caused by defocus or movement blur. To perform this operation will require in general the design of a super-resolution algorithm that integrates a blind deconvolution operation. In this work, the performance of this two-step superresolution method, with performance equal or better than classical MAP superresolution methods, is evaluated on real image sequences with respect to a state of the art BSR method that integrates a blind deconvolution step, to better understand the role played by deconvolution on the global quality provided by super-resolution methods

In Section 2 we present the BSR method based on a regularized energy functional and derive the regularization terms. The alternating minimization scheme and parameter estimation is subsequently described. In section 3 we describe the image SR neural network based method. Finally, Section 4 illustrates applicability of the described methods to real situations.

\section{BLIND SUPERRESOLUTION METHOD}

Blind superresolution (BSR) can find its roots in the related field of multiframe blind deconvolution (MBD). Research on intrinsically MBD methods has begun fairly recently; refer $\mathrm{to}^{2-6}$ for a survey and other references.

Proper registration techniques can suppress large and complex geometric distortions but usually a small between-image shift is still observable. There have been hundreds of methods proposed; see e.g. ${ }^{1}$ for a survey. Here we will assume that registration parameters can be calculated by one of the methods, and if applied, the LR images are registered except to small translations.

Earlier SR approaches assumed that subpixel shifts are estimated by other means. More advanced techniques, such as in, ${ }^{15-17}$ include the shift estimation in the SR process. Other approaches focus on fast implementation, ${ }^{18}$ space-time SR, ${ }^{19} \mathrm{SR}$ with complex image priors for joint image and segmentation estimation, ${ }^{20}$ or SR of compressed video. ${ }^{16}$ Most of the SR techniques assume a priori known blurs. However, in many cases, such as camera motion, blurs can have wild shapes that are difficult to predict; see examples of real motion blurs in. ${ }^{21}$ Authors in ${ }^{22-24}$ proposed BSR that can handle parametric PSFs, i.e., PSFs modeled with one parameter. This 
restriction is unfortunately very limiting for most real applications. $\mathrm{In}^{25}$ we extended our MBD method to BSR in an intuitive way but one can prove that this approach does not estimate PSFs accurately. The same intuitive approach was also proposed in. ${ }^{26}$ To our knowledge, first attempts for theoretically correct BSR with an arbitrary PSF appeared in. ${ }^{27,28}$ The interesting idea proposed therein is to use so-called polyphase components. We adopted the same idea in our formulation. Other preliminary results of the BSR problem with focus on fast calculation are given in ${ }^{29}$ where the authors propose a modification of the Richardson-Lucy algorithm.

In order to solve the BSR problem, i.e, determine the HR image $f$ and volatile PSFs $h_{k}$, we adopt an approach of minimizing a regularized energy function. This way the method will be less vulnerable to noise and better posed. The energy, using vector-matrix notation, consists of three terms and takes the form

$$
E(\vec{f}, \vec{h})=\sum_{k=1}^{K}\left\|\vec{D}_{k} \vec{H}_{k} \vec{f}-\vec{g}_{k}\right\|^{2}+Q(\vec{f})+R(\vec{h}),
$$

where $\vec{h}=\left[\vec{h}_{1}^{T}, \ldots, \vec{h}_{K}^{T}\right]^{T}, \vec{D}_{k}$ represents the discrete case of $\vec{D}$ that includes $\vec{W}_{k}$ and $H_{k}$ denotes a matrix that represents convolution with $\vec{h}_{K}$. The first term measures the fidelity to the data and emanates from our acquisition model Eq. 1. The remaining two are regularization terms that attract the minimum of $E$ to an admissible set of solutions. The form of $E$ very much resembles the energy proposed in ${ }^{7}$ for MBD. Indeed, this should not come as a surprise since MBD and SR are related problems in our formulation. Regularization $Q(\vec{f})$ is a smoothing term of the form

$$
Q(\vec{f})=\alpha \vec{f}^{T} \vec{L} \vec{f},
$$

where $\vec{L}$ is a high-pass filter and $\alpha$ is a positive regularization parameter. A common strategy is to use convolution with the Laplacian for $\vec{L}$, which in the continuous case, corresponds to $Q(f)=\int|\nabla f|^{2}$. Recently, variational integrals $Q(f)=\int \phi(|\nabla f|)$ were proposed, where $\phi$ is a strictly convex, nondecreasing function that grows at most linearly. Examples of $\phi(s)$ are $s$ (total variation), $\sqrt{1+s^{2}}-1$ (hypersurface minimal function), $\log (\cosh (s)$ ), or nonconvex functions, such as $\log \left(1+s^{2}\right), s^{2} /\left(1+s^{2}\right)$ and $\arctan \left(s^{2}\right)$ (Mumford-Shah functional). The advantage of the variational approach is that while in smooth areas it has the same isotropic behavior as the Laplacian, it also preserves edges in images. The disadvantage is that it is highly nonlinear and to overcome this difficulty, one must use, e.g., a half-quadratic algorithm. ${ }^{35}$ For the purpose of our discussion it suffices to state that after discretization we arrive again at Eq. 3, where this time $\vec{L}$ is a positive semidefinite block tridiagonal matrix constructed of values depending on the gradient of $f$. The rationale behind the choice of $Q(f)$ is to constrain the local spatial behavior of images; it resembles a Markov Random Field. Some global constraints may be more desirable but are difficult (often impossible) to define, since we develop a general method that should work with any class of images. Our PSF regularization term $R(\vec{h})$ consists of two terms. The first one is the same smoothing term as for images but applied to blurs, which is a typical prior that penalizes jagged blurs that are rare in real situations. The second term is a consistency term that binds the different volatile PSFs to prevent them from moving freely and unlike the fidelity term (the first term in Eq. 4 it is based solely on the observed LR images. It takes the form of $\|N \vec{h}\|^{2}\left(\mathrm{see}^{30}\right)$. The complete PSF regularization is then given by

$$
R(\vec{h})=\beta \vec{h}^{T} \vec{L} \vec{h}+\gamma\|N \vec{h}\|^{2}
$$

where $\beta$ and $\gamma$ are positive regularization parameters that give different weights to the terms.

\subsection{Alternating minimization}

The complete energy function reads

$$
E(\vec{f}, \vec{h})=\sum_{k=1}^{K}\left\|\vec{D}_{k} \vec{H}_{k} \vec{f}-\vec{g}_{k}\right\|^{2}+\alpha \vec{f}^{T} \vec{L} \vec{f}+\vec{h}^{T}\left(\beta \vec{L}+\gamma N^{T} N\right) \vec{h} .
$$

To find a minimizer, we perform alternating minimizations (AM) of $E$ over $\vec{f}$ and $\vec{h}$. The advantage of this scheme lies in its simplicity. Each term of Eq. 5 is quadratic and therefore convex (but not necessarily strictly convex) 
and the derivatives w.r.t. $\vec{f}$ and $\vec{h}$ are easy to calculate. This AM approach is a variation on the steepest-descent algorithm. The search space is a concatenation of the blur subspace and the image subspace. The algorithm first descends in the image subspace and after reaching the minimum, i.e., $\nabla_{\vec{f}} E=0$, it advances in the blur subspace in the direction $\nabla_{\vec{h}} E$ orthogonal to the previous one, and this scheme repeats. In conclusion, starting with some initial $\vec{h}^{0}$ the two iterative steps are:

step 1.

$$
\vec{f}^{m}=\arg \min _{\vec{f}} E\left(\vec{f}, \vec{h}^{m}\right) \Leftrightarrow \text { solve for } \vec{f}\left(\sum_{k=1}^{K} \vec{H}_{k}^{T} \vec{D}_{k}^{T} \vec{D}_{k} \vec{H}_{k}+\alpha \vec{L}\right) \vec{f}=\sum_{k=1}^{K} \vec{H}_{k}^{T} \vec{D}_{k}^{T} \vec{g}_{k}
$$

step 2 .

$$
\vec{h}^{m+1}=\arg \min _{\vec{h}} E\left(\vec{f}^{m}, \vec{h}\right) \Leftrightarrow \text { solve for } \vec{h}\left(\left[\vec{I}_{K} \otimes \vec{F}^{T} \vec{D}_{k}^{T} \vec{D}_{k} \vec{F}\right]+\gamma N^{T} N+\beta \vec{L}\right) \vec{h}=\left[\vec{I}_{K} \otimes \vec{F}^{T} \vec{D}_{k}^{T}\right] \vec{g},
$$

where $\vec{F}$ represents the convolution with f, $\vec{g}:=\left[\vec{g}_{1}^{T}, \vec{g}_{K}^{T}\right]^{T}$, $\otimes$ is the Kronecker product, $\vec{I}_{K}$ the identity matrix of size $\mathrm{k}$ and $m$ is the iteration step. Note that both steps consist of simple linear equations.

Energy $E$ as a function of both variables $\vec{f}$ and $\vec{h}$ is not convex due to coupling of variables via convolution in the first term of Eq. 5. Therefore, it is not guaranteed that the BSR algorithm reaches the global minimum, instead, one may get trapped in local minima. In our experience, convergence properties improve significantly if we add feasible regions for the HR image and PSFs specified as lower and upper bounds constraints. To solve step 1, we use the method of conjugate gradients (function cgs in Matlab) and then adjust the solution $\vec{f}^{m}$ to contain values in the admissible range, typically, the range of values of $\vec{g}$. It is common to assume that PSF is positive $\left(h_{k} \geq 0\right)$ and that it preserves image brightness. We can therefore write the lower and upper bounds constraints for PSFs as $0 \leq h_{k} \leq 1$. In order to enforce the bounds in step 2, we solve step 2 as a constrained minimization problem (function fmincon in Matlab) rather than using the projection as in step 1. Constrained minimization problems are more computationally demanding but we can afford it in this case since the size of $\vec{h}$ is much smaller than the size of $\vec{f}$.

Parameters $\alpha, \beta$ and $\gamma$ depend on the level of noise. If noise increases, $\alpha$ and $\beta$ should increase, and $\gamma$ should decrease. One can prove that $\alpha$ and $\beta$ are proportional to $\sigma_{n}^{2}$, which is the noise variance. Estimation techniques, such as cross-validation ${ }^{22}$ or expectation maximization, ${ }^{37}$ can be used to determine the correct weights. However, we did not want to increase complexity of the problem any further and thus we set the values in experiments manually according to our visual assessment. If the iterative algorithm begins to amplify noise, we have underestimated the noise level. On contrary, if the algorithm begins to segment the image, we have overestimated the noise level.

\section{NEURAL-NETWORK BASED SUPERRESOLUTION}

In this section the two steps of a neural network based superresolution method are described. In the first step, scattered-point interpolation is performed on projected sequence data using a neural network architecture that learns from examples optimum distance-to-weight interpolation functions for several input sequence noise levels. The second step restores on the interpolated image the combined degradations due to low-resolution optics and detector blurs, as well as residual degradations due to the interpolation procedure. The restoration filter coefficients are obtained by application of a learning process on a set of synthetically generated sequences, for which the superresolution result is readily available. Despite the simplicity of the method, which allows a near real-time implementation, the use of optimum coefficients for both steps of the process enables to obtain results of quality equal or better than classical MAP superresolution methods, with a reduction in computation time by a factor of almost 300 . The reader is referred $t^{38}$ for implementation and evaluation details concerning this method. 


\subsection{Step 1: scattered-point interpolation}

In the scattered-point interpolation phase, sequence pixels are projected on the high-resolution image frame, and image values at the grid nodes are estimated using a scattered-point interpolation technique. This projection operation requires the knowledge with accuracy higher than the input pixel size of the geometrical transforms that link sequence frames. In our method, we have used to this end a sub-pixel registration procedure ${ }^{39}$ based on an iterative gradient descent technique which, in turn, is a development based on the Lucas-Kanade registration procedure. ${ }^{40}$

Using this registration procedure, sub-pixel accurate mappings are computed between the sequence frame selected as reference and the rest of frames in the sequence. The coordinate system of the high-resolution image is taken aligned to that of the reference frame, so that pixels in both images are related by a simple scaling operation. Composing this scaling with the affine transformations determined as a result of registration, a mapping between the high-resolution (HR) image and each of the input sequence frames could be established.

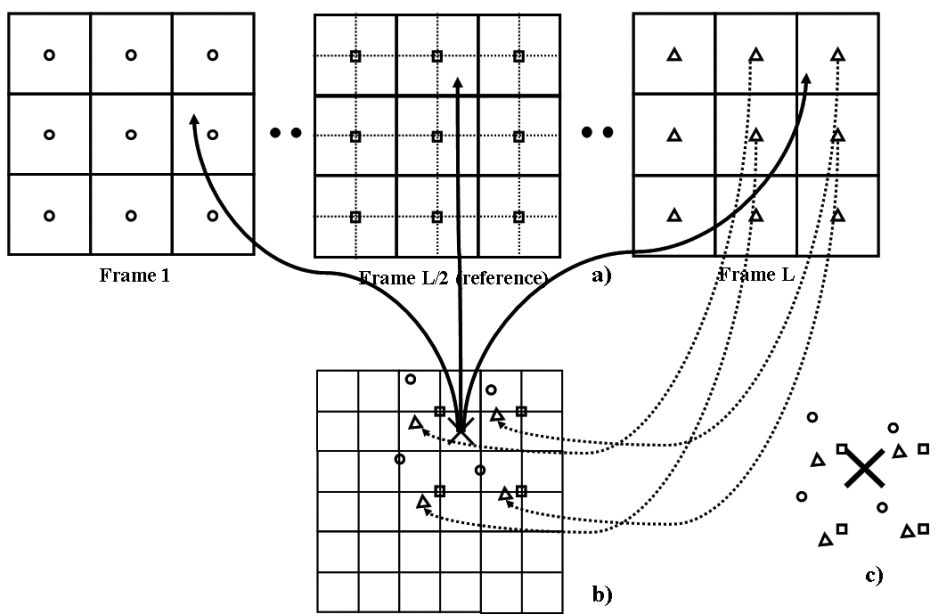

Figure 2: Diagram of the interpolation procedure: a) Sequence frames, with the central frame selected here as reference; b) projection of a HR grid node (zoom $\mathrm{x} 2$ in this example, aligned to the reference frame) onto the sequence frames, and retro-projection of first-order neighborhoods around the projected node location; c) image values at HR grid nodes are estimated by scattered-point interpolation of the retro-projected image values.

Using these mappings, we are able to project a node of the HR grid onto any sequence frame, and the four first-order neighbours of the projected position in the frame low-resolution (LR) rectangular grid can be subsequently identified. Retro-projecting these neighbours onto a common coordinate frame, such as the one defined by the reference frame, and performing this process over the entire sequence, we obtain an irregular cloud of LR image samples that encircle each node of the HR grid. Using these samples, the image value at the HR node is estimated using a scattered-point interpolation technique. A diagram of the interpolation procedure is depicted in figure 1. The scattered-point interpolation is carried out using a modification of the probabilistic neural network (PNN), a neural architecture introduced by Specht. ${ }^{41}$ The PNN is a multivariate kernel density estimator with fixed kernel width. This technique is closely related to a non-parametric regression technique, the Nadaraya-Watson estimator, ${ }^{44}$ and to probability density estimation methods, such as the Parzen windows ${ }^{43}$ method.

A diagram of the proposed neural architecture is presented in figure 2. The network estimates the image value at a HR grid node as a weighted average of the pixels that constitute the first-order neighborhoods of the projected node location at the different sequence frames. The first network layer is composed of a set of identical 


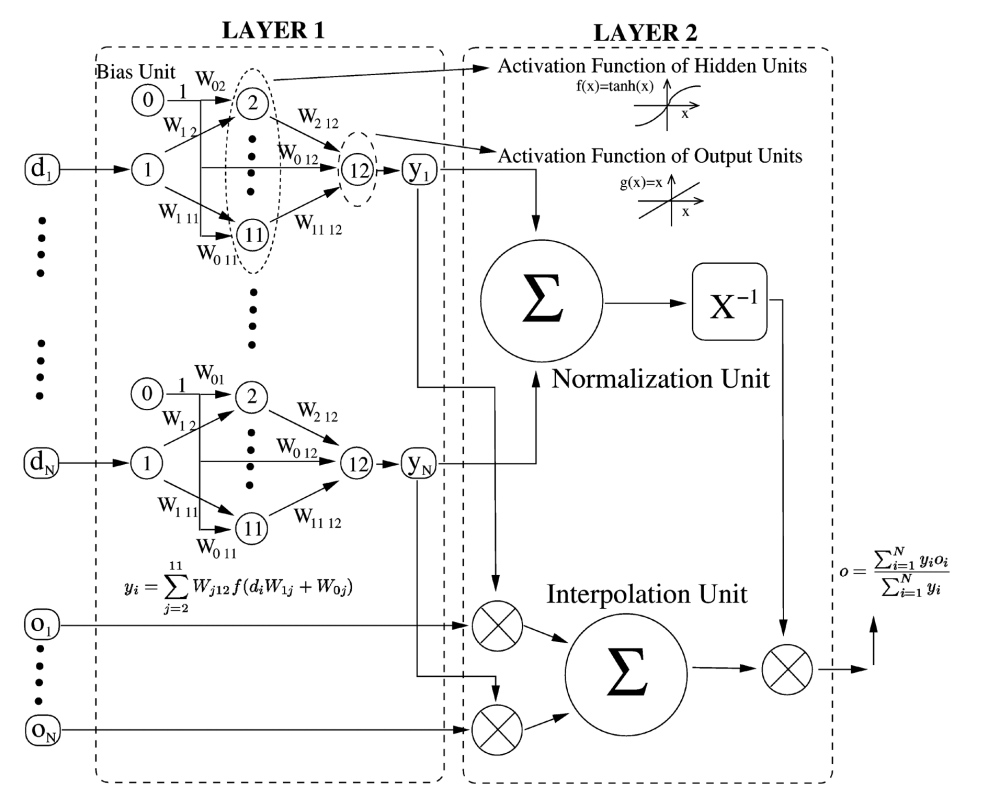

Figure 3: Hybrid MLP-PNN neural architecture for scattered-point image sequence interpolation. The network estimates the image value at a HR grid node as a weighted average of the pixels $(\mathrm{o} 1, \mathrm{oN})$ that constitute the first-order neighborhoods of the projected node location at each sequence frame. First layer MLP networks compute pixel interpolation weights as a non-linear function of the distance to the HR grid node. Second layer units compute the average of weighted pixel values to estimate the image value at the HR node location.

units, four per sequence frame. Each unit receives as input the distance of a retro-projected sequence pixel to the HR grid node, computing its interpolation weight as a non-linear function of this distance. The second network layer contains two summation units to integrate, respectively, weighted pixel contributions and pixel weights. Normalization of sum of weighted pixel contributions by sum of weights provides the final interpolation value. Each HR node is handled independently in this process, making it highly amenable to parallel implementation. In the above procedure, the selection of a kernel shape and width has a significant impact on the quality of the results. Specht $t^{41}$ proposed several function kernels, including the exponential, to be considered for application. The kernel width of a PNN is commonly obtained by a trial-and-error procedure. A too narrow kernel will typically lead to spiky, noisy estimates of the function to be approximated. On the other hand, a too large kernel width will provide an excessive degree of smoothing, sweeping out the function details.

In our system, the kernel function is optimally determined for the image sequence scattered-point interpolation task by a multi-layer perceptron (MLP) ${ }^{44}$ constituting the core of PNN first-layer units. The MLP weights, identical for all units, have been determined by training the full neural architecture on a set of synthetically generated image sequence data, where target interpolation values are readily available. Assuming continuity, the interpolation kernel can be approximated by a two-layer perceptron with a sufficient number of hidden units. ${ }^{45}$ For this purpose, we have used a two-layer perceptron with 10 hidden units and hyperbolic tangent activation functions in the first layer, and a single unit with a linear activation function in the output layer. The adequacy of the hidden layer size to represent the problem complexity was experimentally confirmed by training a network with a substantially larger hidden layer (25 units) and verifying the excellent stability of the obtained kernel solutions. The MLP-PNN network has been trained on a data set composed of a number of independent training patterns, each of them representing an input-output scattered-point interpolation example for the network. In our framework, training patterns are generated synthetically from reference still images that are taken to represent a very high-resolution approximation of a continuous, degradation-free image. Using these images, the output of an imaging sensor of a given (lower) resolution could be simulated by adequate blurring and downsampling operations. This procedure can be carried out to generate synthetic LR image sequence data, together with the 
sought output for the two steps of our super-resolution process. Gaussian noise could be added ${ }^{46}$ to account for several sources of noise in the system. Varying the amount of Gaussian noise added to the input LR sequences, the network could be trained to operate with imagery of different noise levels. In our implementation, we have used as reference images a set of 25 visible-range, high-resolution satellite images of urban landscape acquired with the Quickbird system. ${ }^{47}$ Figure 4 presents some samples extracted from this image set.

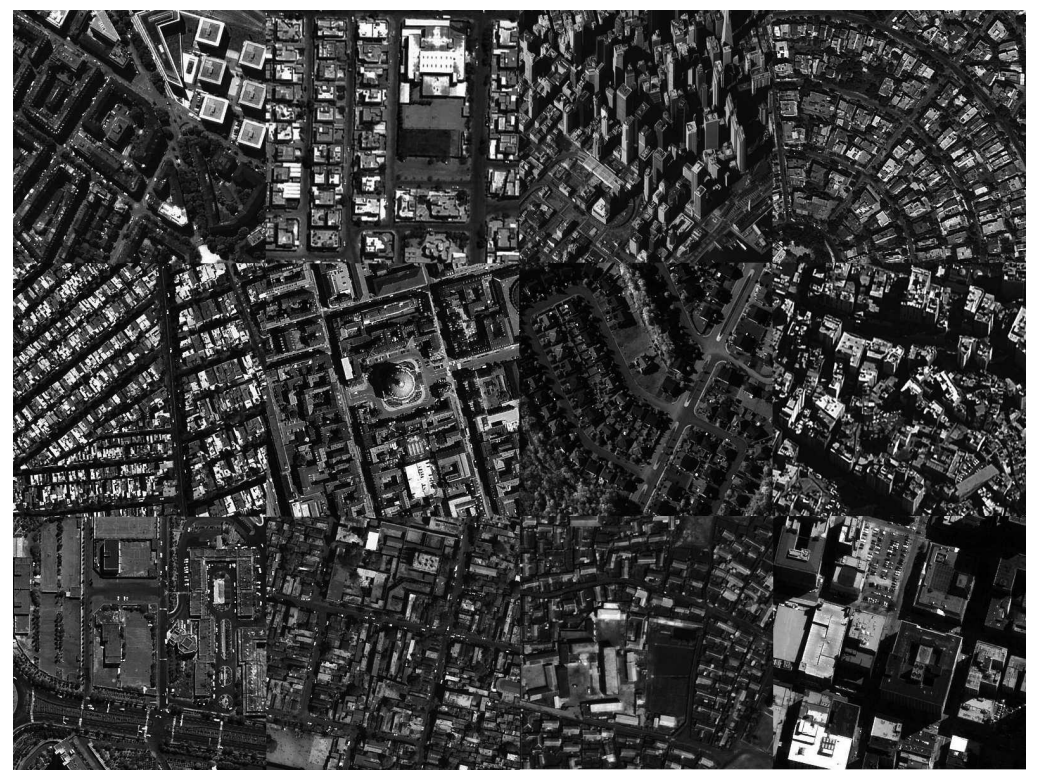

Figure 4: Composition of image samples of the reference image set, containing Quickbird satellite high-resolution images of urban scenes.

In our architecture, MLP units compute the interpolation weight of a given image sample as a non-linear function of its distance to the target location. Once the learning phase has concluded, the MLP could be used to generate a look-up table relating distance to interpolation weight. In the deployment phase, we have replaced MLP units in our architecture with corresponding distance-to-weight look-up tables, with distance sampled at 10-3 pixel intervals. This has provided a very significant speed-up of the process with a negligible loss in performance. Presented PNN interpolation results have been obtained in all cases with MLP generated look-up tables. In figure 5 are presented graphs of the distance-to-weight PNN kernel functions obtained by training our neural architecture on data sets generated using the reference image set, with input data degraded by Gaussian noise of several standard deviations ( $\sigma=1,5,10$ and 20 gray-levels). In all cases, the network was trained on 25 -frame sequences, corresponding to a 1s video stream in the European video standard. Obtained results show a steady increase in kernel width with input data noise. Intuitively, at low noise levels preservation of image detail is a primary factor, and kernel widths are adjusted to impose object continuity on the estimated image, avoiding jagged edges typical of nearest-neighbour interpolation. At higher levels, noise becomes a leading factor in the reconstruction error, and kernels widen correspondingly to smooth the results, at the cost of some loss in detail preservation. The training algorithm performs these function shape modifications in an autonomous, data-driven manner.

The performance of the proposed learning-based procedure has been compared to other scattered-point interpolation methods proposed in the super-resolution literature ${ }^{48-50}$ with excellent results. The reader is referred to $^{38}$ for detailed results of this comparison.

\subsection{Step 2: pixel size blurring restoration}

As a result of the previous processing steps, an intermediate image sampled at the high-resolution rate is obtained. This image is still degraded at least by low-resolution optics and detector blurs, as well as by any residual errors of the interpolation procedure. As interpolation only partially filters sequence noise, this intermediate image is 


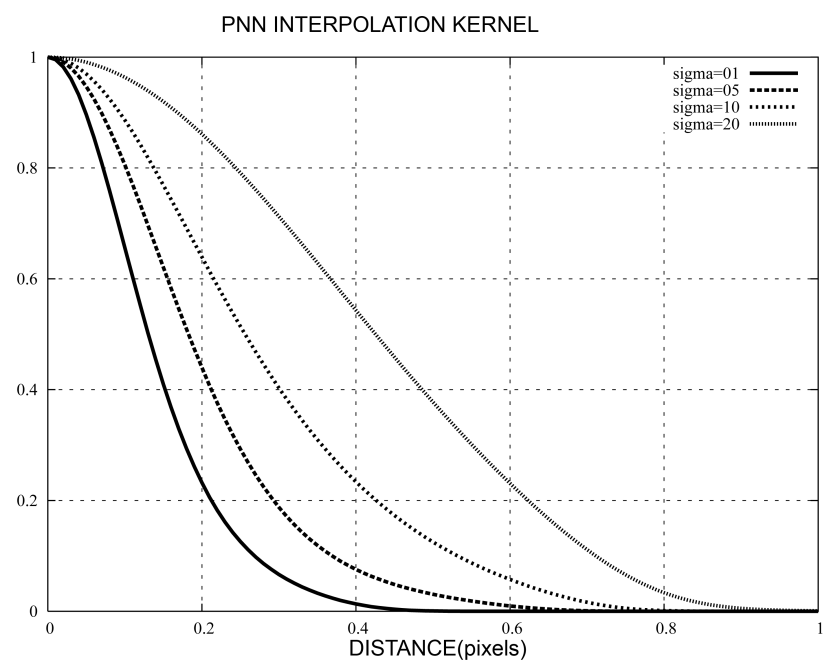

Figure 5: Optimal distance-to-weight PNN kernel functions for different noise variances $(\sigma=1,5,10,20$ graylevels), as learned by neural networks trained on samples extracted from the reference image set. In all cases, networks were trained on 25-frame sequences. Distance is measured in units of LR sequence pixel size.

also affected by some level of possibly coloured noise. Wiener filtering ${ }^{51}$ is one of the preferred choices to perform the restoration step in two-step super-resolution methods. ${ }^{49,52}$ These linear restoration filters are optimum on RMS sense, but require knowledge of the degradation transfer function, $\mathrm{H}$, and the power spectra of the deblurred image $S_{f}$, and noise $S_{\eta}$. In the Fourier domain, the Wiener filter is given by the following expression: ${ }^{51}$

$$
W\left(f_{x}, f_{y}\right)=\frac{H^{*}\left(f_{x}, f_{y}\right)}{\mid H\left(f_{x},\left.f_{y}\right|^{2}+S_{\eta}\left(f_{x}, f_{y}\right) / S_{f}\left(f_{x}, f_{y}\right)\right.} \approx \frac{H^{*}\left(f_{x}, f_{y}\right)}{\mid H\left(f_{x},\left.f_{y}\right|^{2}+K\right.},
$$

where * denotes here complex conjugates. In practice $[11,13]$, the power spectra quotient in the denominator could be replaced by a suitably defined constant, $K$, with only moderate drops in performance. In our image formation model, degradation is due to the combined action of three processes that act incoherently: lens blur, detector blur and residual degradations caused by interpolation. According to linear system theory, ${ }^{53}$ the degradation OTF could be obtained by point-wise multiplication of the OTF's corresponding to these three processes:

$$
H=O T F_{\text {deg }}\left(f_{x}, f_{y}\right)=O T F_{\text {opt }}\left(f_{x}, f_{y}\right) . O T F_{\text {det }}\left(f_{x}, f_{y}\right) . O T F_{\text {interp }}\left(f_{x}, f_{y}\right),
$$

The first two transfer function could be approximated, respectively, by the standard diffraction and detector aperture OTF's. ${ }^{53}$ On the other hand, as analytical modelling of scattered-point interpolation transfer functions is a difficult task, these effects are usually left out of the degradation model. Consequently, restoration does not attempt to correct any degradations caused by the previous interpolation step. This decoupling between the interpolation and restoration steps of the super-resolution procedure is a common criticism of two-step methods. ${ }^{54}$

In our approach, restoration is performed using linear filters in the spatial domain. Filter coefficients are determined by means of a learning process based on examples, to obtain minimum squared error estimates of HR images when applied to intermediate images obtained by the scattered-point interpolation step. As interpolated images are used as input, learning implicitly considers residual degradations caused by interpolation, effectively coupling both steps of the super-resolution method. Spatial linear filtering is performed by convolving the interpolated image with a 2-D mask optimized for this problem. The number of filter coefficients to be determined has been drastically reduced by imposing isotropy on the filter shape, with values of filter coefficients depending solely on their distance to the filter center. The optimal filters have been obtained by minimizing a sum-of-squares 
error function, using the pseudoinverse method. ${ }^{46}$ To avoid over-fitting of filter coefficients, only rather lowdimensional filters have been considered. These filters have been trained with large datasets containing around 1.1x107 input-output examples of the required filter operation, to obtain a largely over-determined estimation problem. As a result of the training procedure, to obtain a superresolution zoom factor of 3 , symmetric filters of size 9x9 have been selected for use for all noise levels but the highest, where a filter of size 7x7 is used instead. These filters have 15 and 10 different coefficients, respectively. For these relatively low-dimensional problems, minimized on large training datasets, the sum-of-squares error function has shown to provide acceptable results as has been check experimentally by comparison with filter solutions obtained with error functions incorporating regularization terms. In figure 5 are presented the determined restoration filter coefficients for the four noise levels and a filter size of 9x9 pixels, corresponding to 15 different symmetric coefficients. For small input noise levels, the obtained filters show a marked oscillatory behaviour typical of high-frequency enhancement operations. As noise grows, the oscillations decrease in number and amplitude, as smoothing becomes an increasingly dominant operation. All these modifications have been carried out autonomously by our training procedure, driven by minimization of an error function. The training datasets have also been used to optimally fit a Wiener filter adapted to correct the lens and detector combined degradation. Considering the simplified expression in Eq. (8), this involves fitting the value of the $K$ parameter to minimize the sum-of-squares error between the filtered input and the output of the examples in the corresponding dataset. This minimization has been carried out using a standard uni-dimensional golden section search. ${ }^{46}$ This fitting introduces some coupling between the interpolation and restoration steps of the process, as the $K$ parameter is fixed to minimize Wiener restoration errors on images generated by a precise interpolation procedure. A complete coupling will require, nevertheless, a proper modeling of the interpolation effects both in terms of transfer function and noise filtering. These models are implicitly learned from the input-output training set by our spatial filtering scheme with free parameters.
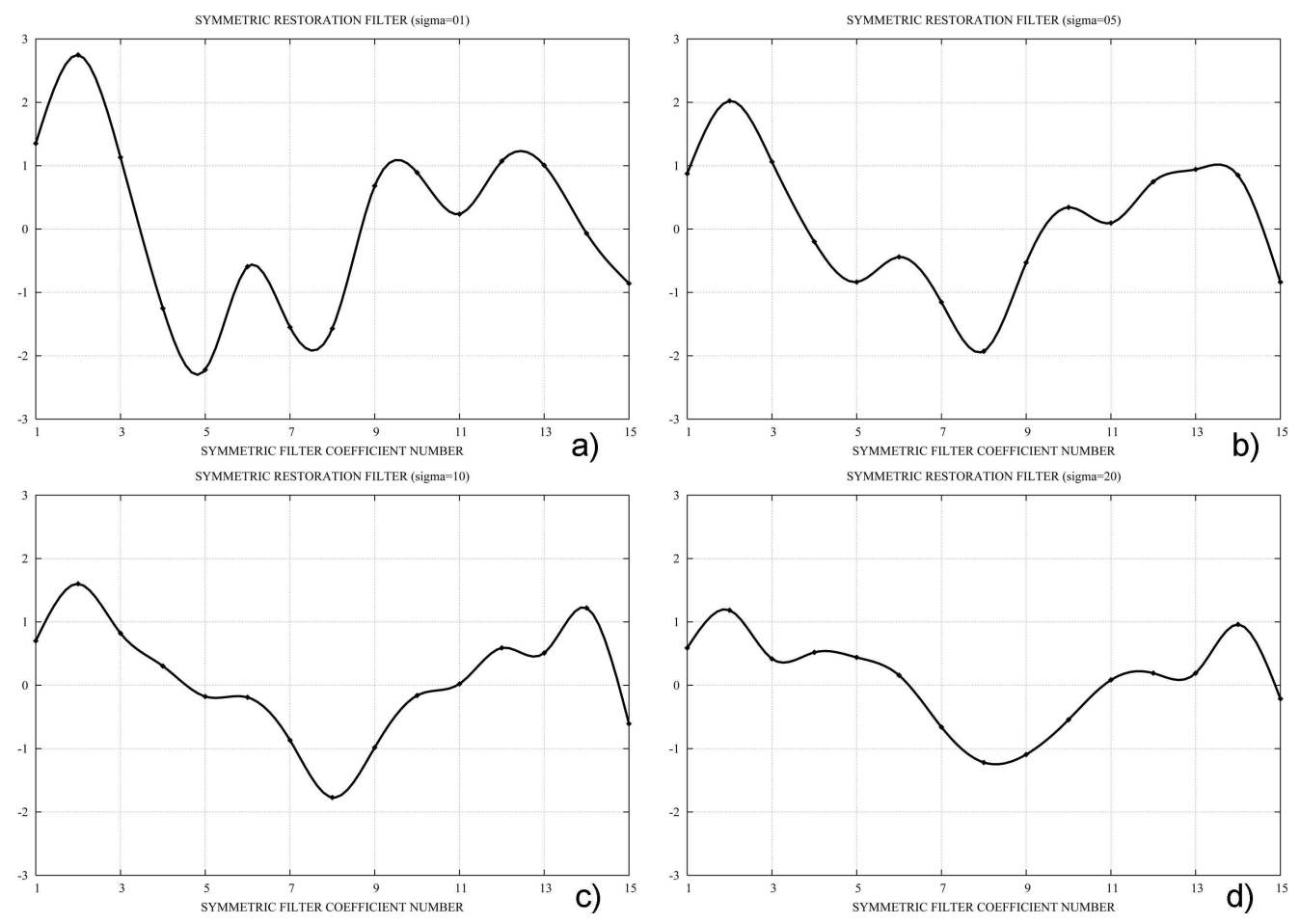

Figure 6: Symmetric coefficients of optimal 9x9 restoration filters for a superresolution zoom factor of 3, and the four noise levels considered: a) $\sigma=1$; b) $\sigma=5$; c) $\sigma=10$ and d) $\sigma=20$ gray-levels.

In figure 7 are presented restorations obtained with filters of both types when processing a MLP-PNN interpolated image of an USAF 1951 test target viewgraph, which was part of a laboratory setup with a mixture of objects of different type. Figure 7-a shows the image obtained after application of the first step of our 
method. In this image is clearly visible the structure of the horizontal and vertical tri-bar patterns located at the upper-leftmost side of the picture. This fine structure was completely washed out by application of a Wiener filter adapted only to lens and detector degradations, as can be seen in figure 7-b. Contrariwise, application of the obtained spatial linear filter, with coefficients optimized to take also into account residual interpolation degradations, maintained and enhanced the structure in those patterns. Other differences between both restorations are visible on the numbers at the rightmost side, which are clearly more blurred in Wiener filter restoration results.

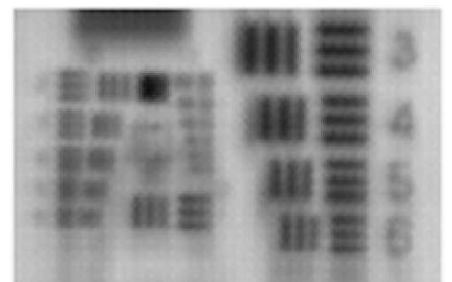

a)

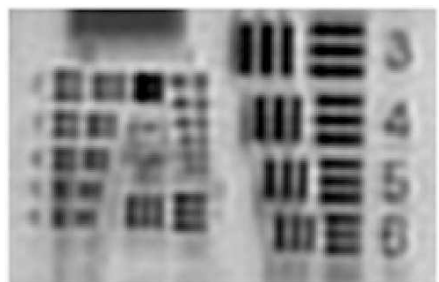

b)

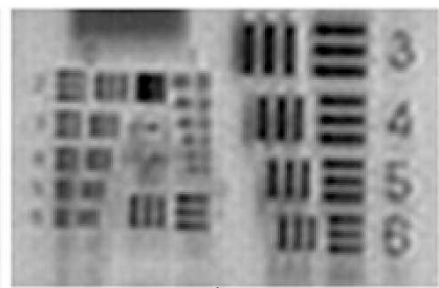

c)

Figure 7: Improvement obtained by taking into account interpolation degradations on the restoration filter design: a) result of the first-step of super-resolution; b) restoration of the image in a) with a Wiener filter for combined optical and detector degradations and c) restoration obtained with a spatial linear filter with learned coefficients. Restoration with this last filter enhanced the structure of tri-bar patterns at the upper-leftmost side of the picture, while this fine structure was washed out by application of a Wiener filter adapted only to lens and detector degradations.

\section{EXPERIMENTS}

The following experiments with the described methods aim to first compare performance with other techniques and second demonstrate its applicability to real scenarios with misregistered input images.

\subsection{Real data}

The first experiment shows the main characteristics of the BSR method. Fig 8 shows a frame sequence taken from the Vanderwalle dataset ${ }^{55}{ }^{*}$. It is worth to mention that in the LR frames (Fig. 8a) a significant amount of spectral aliasing is present. We will take advantage of such aliasing to recover high frequencies in the HR frames. Fig $8 \mathrm{c}$ shows the edge information taken as the prior by the BSR method to regularize the solution. Fig. 8d shows that this method is able to provide the PSF of each frame as a byproduct.

The next example corresponds to a frame sequence from the Farsiu\&Milanfar database ${ }^{18}{ }^{\dagger}$. Fig. 9a shows one of the original "eia" frames and Fig. 9b shows the BSR result. For the next example, we took a Olympus C5050Z digital camera and captured four photos, registered them with cross correlation and cropped each to 70x40 pixels (Fig. 9c). Fig. 9d shows the BSR result. A potential pitfall that we have to take into consideration is a feasible range of SR factors. As the SR factor increases we need to take more LR images and the stability of BSR decreases. Hence we limit ourselves the SR factor between 1 and 2.5 in most practical applications.

The second group of experiments illustrates the influence of the number of iterations in the reconstruction provided by the BSR technique. We have taken the "alpaca" dataset from the Farsiu\&Milanfar database and reconstructing the HR frames for different iterations (Fig. 10). It turns out that the best reconstruction results appear for a reduced number of iterations ( 2 in this particular example). This result can be corroborated both visually and also using a non-reference metric proposed by the authors in. ${ }^{56}$ A similar response was observed in the "castle" example illustrated in Fig. 8. Fig. 11 shows another example corresponding to a license plate identification scenario. Here the BSR result provides the best reconstruction against other interpolation techniques. The previously cited non-reference metric corroborates this result (Fig. 11d). Fig. 12 illustrates

\footnotetext{
*http://lcavwww.epfl.ch/software/superresolution/index.html

${ }^{\dagger}$ http://www.soe.ucsc.edu/ milanfar/DataSets/
} 
the BSR performance in a face recognition experiment in comparison with the result provided by a classical interpolation technique. It is worth noting how the BSR method is able to recover the high frequency contents extracted from the LR input sequence (see Fig. 12c\&12f) as opposed to the result provided by the interpolation technique which is unable to recover such information (see Fig. 12b\&12e).

A third experiment shows the performance of the MLP-PNN method. Fig. 13 presents the reconstruction results after applying the first and second step of the described method and includes a comparison with a bicubic interpolation method.

A final group of experiments provides a comparative study of the performance of different SR methods. Here is left to the reader to decide which method provide the best reconstruction quality (Fig. 14).

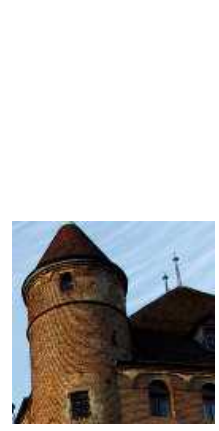

(a)

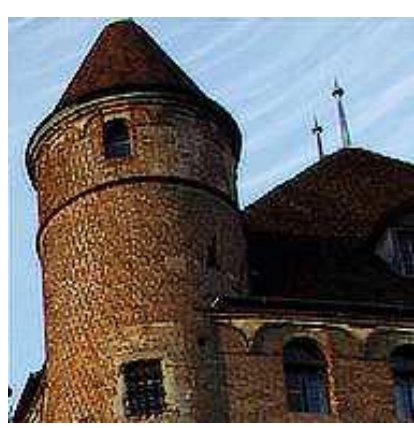

(b)

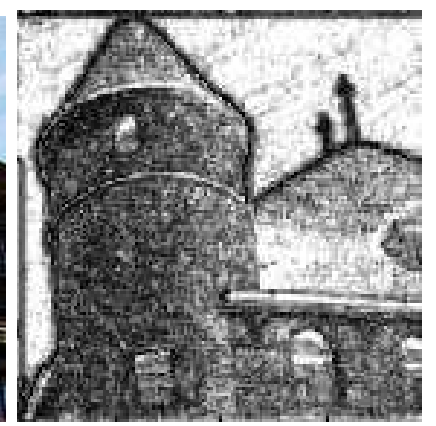

(c)

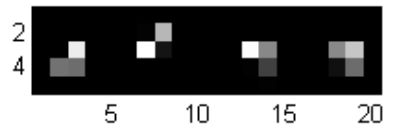

(d)

Figure 8: (a) Shown is one of four LR frames. Frame sequence was taken from Vanderwalle dataset ${ }^{55}$. Note the presence of aliasing in the brick structure of the tower (b) The BSR result (c) Image of the prior edge information used by the BSR method; (d) PSF of each frame estimated by the BSR method

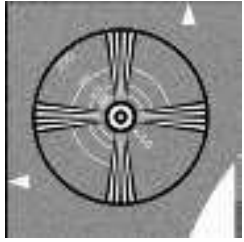

(a)

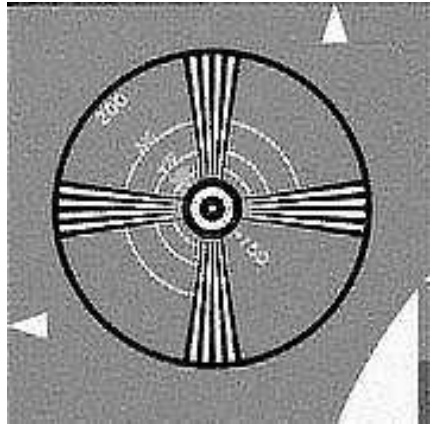

(b)

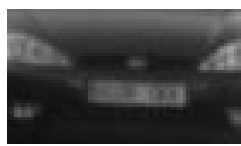

(c)

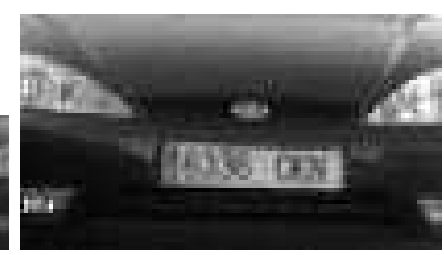

(d)

Figure 9: (a) Shown is one of four LR frames. Note the presence of aliasing in such frames as in the previous example. Frame sequence was taken from Farsiu\&Milanfar "eia" dataset; (b) The BSR result. (c) Shown is one of four LR frames taken by F.S. from a real traffic sequence (d) BSR result

\subsection{Limiting factors}

The main problem that limits the robustness of most of the SR methods are due to the presence of high compression rates which still are omnipresent in most of the video surveillance scenarios. Very recently Pham ${ }^{58}$ has noted that deconvolution is found ineffective by irreversible DCT quantization what produces annoying blocking artifacts after compression. In the next experiment we are interested to analyze the impact of the introduction of different levels of compression in the test sequences. We have taken the Farsiu\&Milanfar "car" sequence and saved as JPEG format through the Matlab command imwrite for different quality levels. Fig. 8 shows that for a compression quality of $80 \%$ or lesser the reconstruction artifacts using the BSR method become unacceptable. 


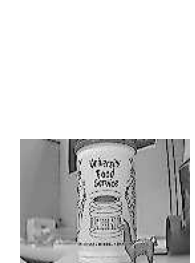

(a)

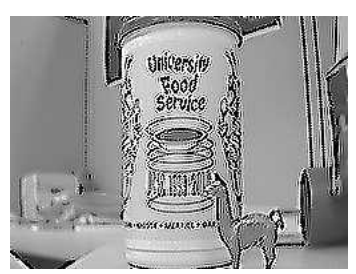

(e)

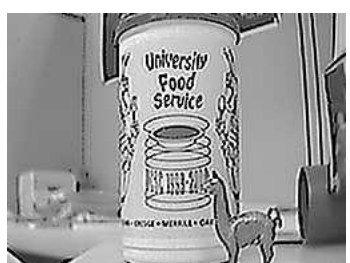

(b)

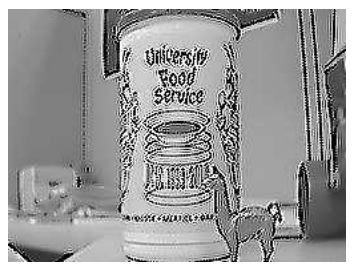

(f)

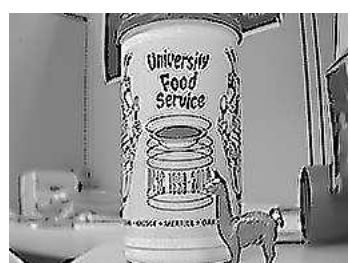

(c)

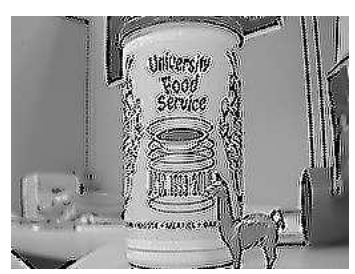

(g)

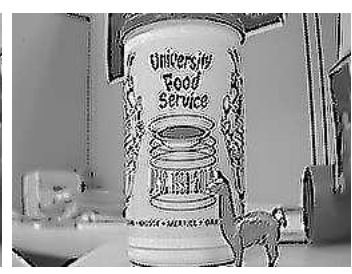

(d)

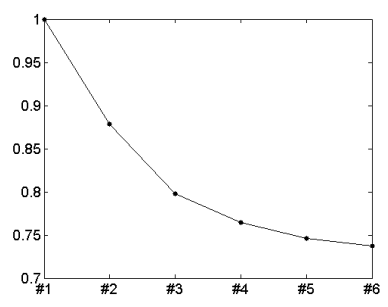

(h)

Figure 10: (a) Shown one of four LR frames from the Farsiu \& Milanfar "alpaca" dataset. (b-g) Different BSR results for two, three, four, five, six and seven iterations respectively; (h) Quality of the reconstruction results using an anisotropic measure. ${ }^{56}$ Note that the best reconstruction corresponds to (b) that matches the visual assessment. Horizontal axis represents reconstruction results and vertical axis represents normalized quality measure.

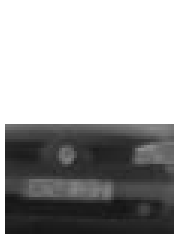

(a)

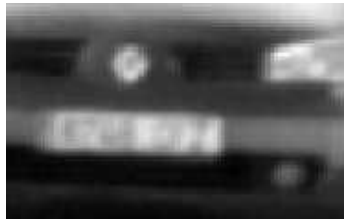

(b)

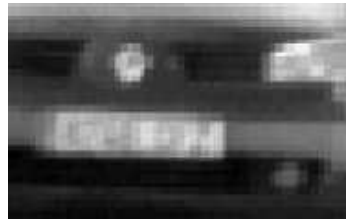

(c)

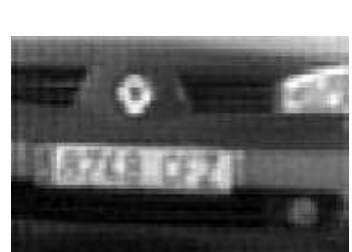

(d)

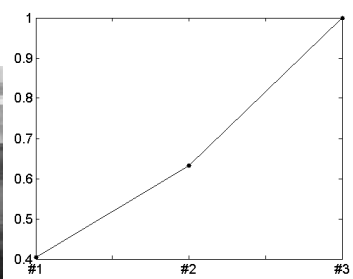

(e)

Figure 11: (a) Shown one of four LR frames taken by F.S. from a traffic sequence; (b) Bilinear interpolation result; (c) Nearest neighbor interpolation result; (d) BSR result; (e) Quality of the reconstruction results using an anisotropic measure. ${ }^{56}$ Horizontal axis represents reconstruction results and vertical axis represents normalized quality measure.

\subsection{Superresolution of a full video}

Superresolution can be extended to a full video sequence by simply shifting along the temporal line (see Fig. 16). Fig. 17 shows an example of a LR video sequence which resolution has been enhanced by the BSR technique described here.

\section{CONCLUSIONS}

This paper has surveyed two state-of-the-art SR methods. First, the BSR method has been proved to be meaningful for cases when insufficient number of input LR images is available to perform SR with only integer factors, such as two or three. To achieve truly robust methodology applicable in real situations, we adopted the regularized energy minimization approach, which we solve by alternating-minimization scheme. The fundamental improvement on previously proposed SR methods is the notion of estimating PSFs in the HR scale, which indirectly aligns LR images with subpixel accuracy. Using registration parameters inside the algorithm instead of registering input images gives better results and paves the way for including methods of making registration parameters more accurate during reconstruction of the HR image. ${ }^{34,57}$ The second method was based on the application of scattered-point interpolation on projected sequence data, followed by a filtering operation to restore 


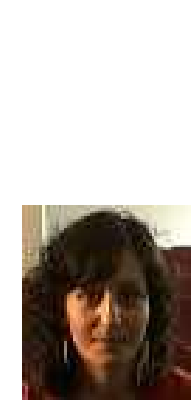

(a)

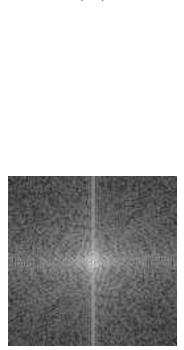

(d)

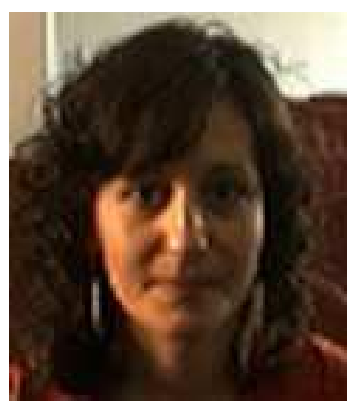

(b)

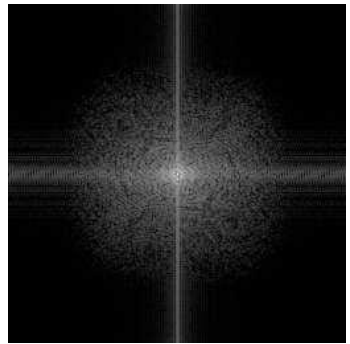

(e)

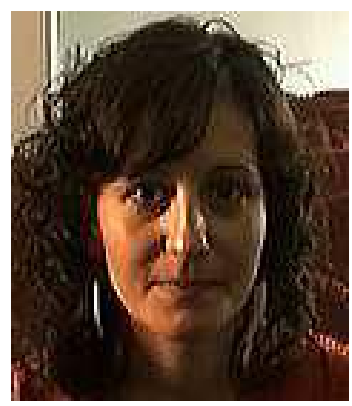

(c)

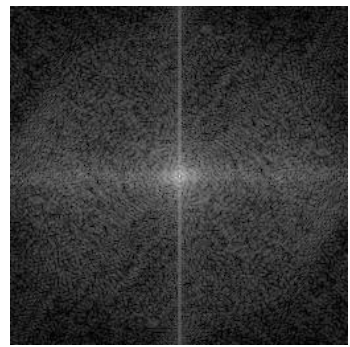

(f)

Figure 12: Superresolution a color video sequence. (a) Shown is one LR frame taken with a webcamera; (b) Interpolated result;(c) BSR result; (d) Magnitude of the FFT of (a); (e) Magnitude of the FFT of (b). Note the loss of high frequencies, (f) Magnitude of the FFT of (c). Note how the BSR technique is able to recover the high frequency part of the spectrum

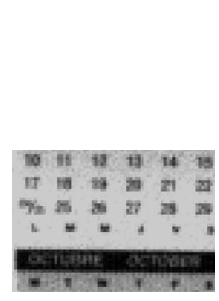

(a)

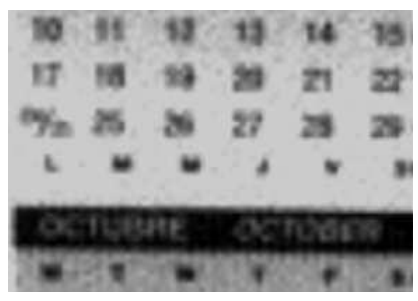

(b)

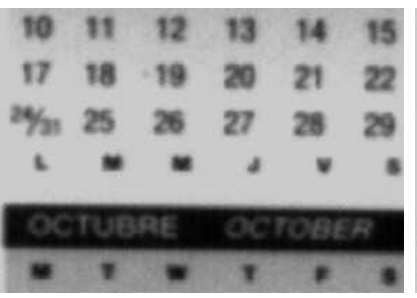

(c)

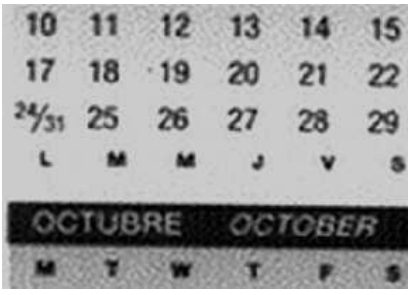

(d)

Figure 13: SR results on the 'calendar' sequence using the MLP-PNN method(a) Shown is one of the LR frames. (b) Bicubic interpolation result (c) Result after applying the first step of the MLP-PNN method; (d) Result after the two-step MLP-PNN method

degradations associated to sequence pixel size and residual errors introduced by interpolation. The advantage of the second technique is that it can be used in quasi-real time applications.

\section{ACKNOWLEDGMENTS}

This research has been partially supported by the following grants: TEC2007-67025/TCM, TEC2006-28009-E, BFI-2003-07276, TIN-2004-04363-C03-03 by the Spanish Ministry of Science and Innovation, and by PROFIT projects FIT-070000-2003-475 and FIT-330100-2004-91. Also, this work has been partially supported by the Czech Ministry of Education under the project No. 1M0572 (Research Center DAR) and by the Czech Science Foundation under the project No. GACR 102/08/1593 and the CSIC-CAS bilateral project 2006CZ0024.

\section{REFERENCES}

[1] Zitová, B. and Flusser, J. (2003) Image registration methods: A survey. Image and Vision Computing, 21, 977-1000. 


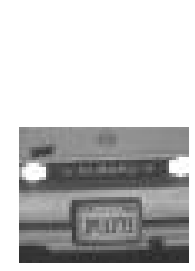

(a)

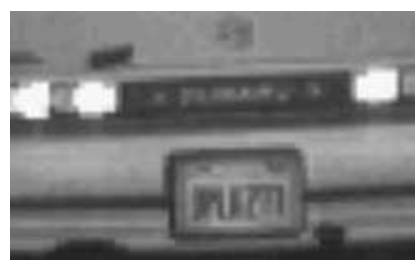

(b)

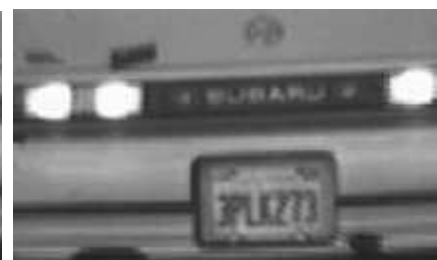

(c)

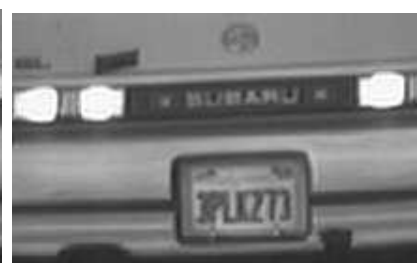

(d)

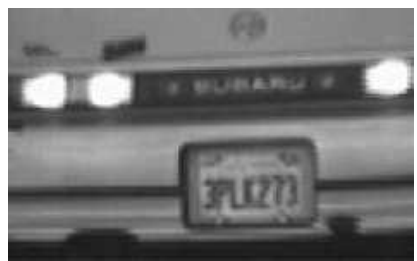

(e)

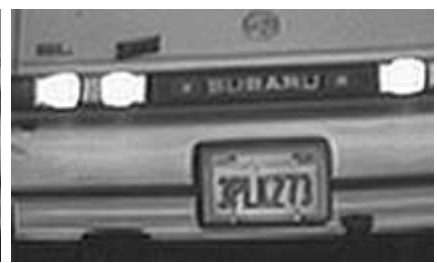

(f)

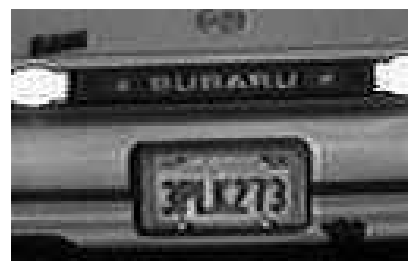

(g)

Figure 14: Superresolution method comparison. (a) Shown is one "car" LR frame from the Farsiu \& Milanfar dataset; (b) bilinear interpolation; (c) result using a preconditioned Tikhonov regularization method; (d) result using a Bayesian method ${ }^{15}$ (e) Farsiu \& Milanfar result ${ }^{13}$ (f) MLP-PNN result (g) BSR result

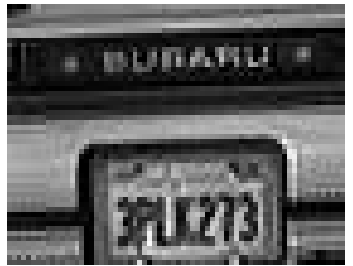

(a)

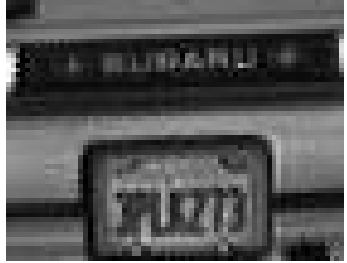

(b)

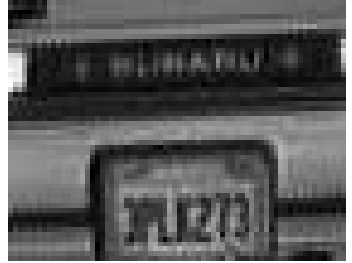

(c)

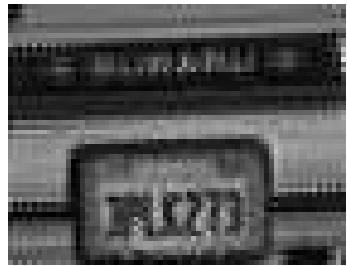

(d)

Figure 15: Influence of the compression factors on the BSR reconstruction quality. (a) Compression quality 100\% (b)Compression quality 90\% (c) Compression quality $85 \%$ (d) Compression quality 80\%. Note that for a compression quality of $80 \%$ and lesser the overall quality is unacceptable although some digits of the license are still recognizable.

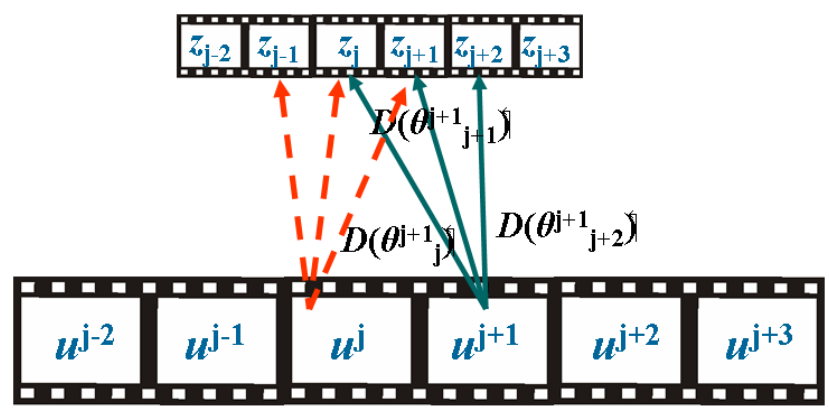

Figure 16: The SR of a full video sequence (u-frames) can be simply obtained by shifting along the temporal line the LR frames (z-frames) to be processed

[2] Harikumar, G. and Bresler, Y. (1999) Perfect blind restoration of images blurred by multiple filters: Theory and efficient algorithms. IEEE Trans. Image Processing, 8, 202-219.

[3] Giannakis, G. and Heath, R. (2000) Blind identification of multichannel FIR blurs and perfect image restoration. IEEE Trans. Image Processing, 9, 1877-1896.

[4] Pai, H.-T. and Bovik, A. (2001) On eigenstructure-based direct multichannel blind image restoration. IEEE Trans. Image Processing, 10, 1434-1446. 


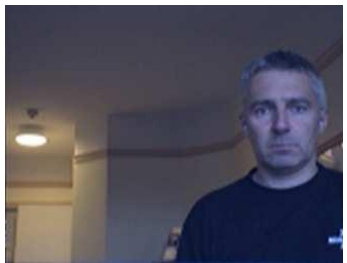

(a)

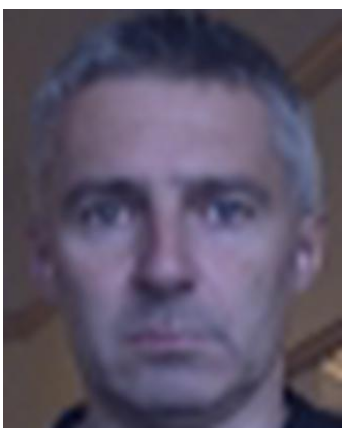

(b)

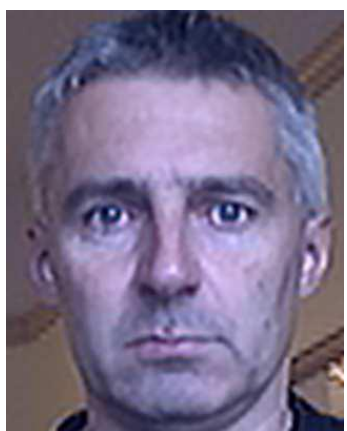

(c)

Figure 17: Superresolution of a full video sequence (frame size 160x120 pixels) (a) Interpolated LR frame; (b) BSR result

[5] Panci, G., Campisi, P., Colonnese, S., and Scarano, G. (2003) Multichannel blind image deconvolution using the bussgang algorithm: Spatial and multiresolution approaches. IEEE Trans. Image Processing, 12, 1324-1337.

[6] Šroubek, F. and Flusser, J. (2003) Multichannel blind iterative image restoration. IEEE Trans. Image Processing, 12, 1094-1106.

[7] Šroubek, F. and Flusser, J. (2005) Multichannel blind deconvolution of spatially misaligned images. IEEE Trans. Image Processing, 14, 874-883.

[8] You, Y.-L. and Kaveh, M. (1999) Blind image restoration by anisotropic regularization. IEEE Trans. Image Processing, 8, 396-407.

[9] Rajagopalan, A. and Chaudhuri, S. (1999) An MRF model-based approach to simultaneous recovery of depth and restoration from defocused images. IEEE Trans. Pattern Analysis and Machine Intelligence, 21, $577-589$.

[10] Park, S., Park, M., and Kang, M. (2003) Multiframe image restoration and registration IEEE Sig. Proc. Magazine, 20, 21-36.

[11] Kim, S.P., Bose, N.K. and Valenzuela, H.M. (1990) Recursive reconstruction of high resolution image from noisy undersampled multiframes IEEE Trans. Acoust. Speech Sig. Proc., 38, Wiley, 1013-1027.

[12] Tsai, R.Y., Huang, T.S. (eds) (1984) Multiframe image restoration and registration in Advances in Computer Vision and Image Processing, 1, Wiley, 317-339.

[13] Farsui, S., Robinson, D., Elad, M., and Milanfar, P. (2004) Advances and challenges in super-resolution. Int. J. Imag. Syst. Technol., 14, 47-57.

[14] Borman, S., Stevenson, R.L. (1998) Superresolution from image sequences- a review Proc. Midwest Symp. Circ. Syst.

[15] Hardie, R., Barnard, K., and Armstrong, E. (1997) Joint map registration and high-resolution image estimation using a sequence of undersampled images. IEEE Trans. Image Processing, 6, 1621-1633.

[16] Segall, C., Katsaggelos, A., Molina, R., and Mateos, J. (2004) Bayesian resolution enhancement of compressed video. IEEE Trans. Image Processing, 13, 898-911.

[17] Woods, N., Galatsanos, N., and Katsaggelos, A. (2006) Stochastic methods for joint registration, restoration, and interpolation of multiple undersampled images. IEEE Trans. Image Processing, 15, 201-213.

[18] Farsiu, S., Robinson, M., Elad, M., and Milanfar, P. (2004) Fast and robust multiframe super resolution. IEEE Trans. Image Processing, 13, 1327-1344.

[19] Shechtman, E., Caspi, Y., and Irani, M. (2005) Space-time super-resolution. IEEE Trans. Pattern Analysis and Machine Intelligence, 27, 531-545.

[20] Humblot, F. and Muhammad-Djafari, A. (2006) Super-resolution using hidden markov model and bayesian detection estimation framework. EURASIP Journal on Applied Signal Processing, 2006, 36971.

[21] Ben-Ezra, M. and Nayer, S. (2004) Notion-based motion deblurring. IEEE Trans. Pattern Analysis and Machine Intelligence, 26, 689-698. 
[22] Nguyen, N., Milanfar, P., and Golub, G. (2001) Efficient generalized cross-validation with applications to parametric image restoration and resolution enhancement. IEEE Trans. Image Processing, 10, 1299-1308.

[23] Woods, N., Galatsanos, N., and Katsaggelos, A. (2003) EM-based simultaneous registration, restoration, and interpolation of super-resolved images. Proceeding of ICIP, Barcelona, September, pp. 303-306. IEEE Computer Society.

[24] Rajan, D. and Chaudhuri, S. (2003) Simultaneous estimation of super-resolved scene and depth map from low resolution defocused observations. IEEE Trans. Pattern Analysis and Machine Intelligence, 25, 11021117.

[25] Šroubek, F. and Flusser, J. (2006) Resolution enhancement via probabilistic deconvolution of multiple degraded images. Pattern Recognition Letters, 27, 287-293.

[26] Chen, Y., Luo, Y., and Hu, D. (2005) A general approach to blind image super-resolution using a PDE framework. Proceedings of SPIE, Beijing, July, pp. 1819-1830. SPIE.

[27] Wirawan, Duhamel, P., and Maitre, H. (1999) Multi-channel high resolution blind image restoration. Proceedings of ICASSP, Phoenix, AR, March, pp. 3229-3232. IEEE Computer Society.

[28] Yagle, A. (2003) Blind superresolution from undersampled blurred measurements. Proceedings of SPIE, Bellingham, December, pp. 299-309. SPIE.

[29] Biggs, D., C.L.Wang, Holmes, T., and Khodjakov, A. (2004) Subpixel deconvolution of 3D optical microscope imagery. Proceedings of SPIE, Denver, CO, October, pp. 369-380. SPIE.

[30] Šroubek, F., Cristóbal, G., and Flusser, J. (2007) A unified approach to superresolution and multichannel blind deconvolution. IEEE Trans. Image Processing, 16, 2322-2332.

[31] Lin, Z. and Shum, H.-Y. (2004) Fundamental limits of reconstruction-based superresolution algorithms under local translation. IEEE Trans. Pattern Analysis and Machine Intelligence, 26, 83-97.

[32] Pelletier, S. and Cooperstock, J. (2007) Fast super-resolution for rational magnification factors. Proceedings of ICIP, San Antonio, TX, September, pp. 65-68. IEEE Computer Society.

[33] Šroubek, F., Flusser, J., and Cristóbal, G. (2007) Multiframe blind deconvolution coupled with frame registration and resolution enhancement. In Campisi, P. and Egiazarian, K. (eds.), Blind Image Deconvolution: Theory and Applications. CRC Press, FL.

[34] Šroubek, F., Flusser, J. and Cristóbal, G. (2007) Superresolution and blind deconvolution for rational factors. The Computer Journal, in press.

[35] Aubert, G. and Kornprobst, P. (2002) Mathematical Problems in Image Processing. Springer Verlag, New York.

[36] Tschumperlé, D. (2002) PDE's Based Regularization of Multivalued Images and Applications. PhD thesis University of Nice-Sophia Antipolis.

[37] Molina, R., Vega, M., Abad, J., and Katsaggelos, A. (2003) Parameter estimation in Bayesian high-resolution image reconstruction with multisensors. IEEE Trans. Image Processing, 12, 1655-1667.

[38] Miravet, C. and Rodriguez, F.B. (2007) A two step neural network based algorithm for fast image superresolution Image and Vision Computing, 25, 1499-1473.

[39] Irani, M. and Peleg, S. (1991) Improving resolution by image registration CVGIP: Graph Model Img. Proc., 53, 231-239.

[40] Lucas, B.D. and Kanade, T. (1981) An iterative image registration technique with an application to stereo vision Int. J. Conf. Artif. Intell., 674-679.

[41] Specht, D.F. (2002) Neural Networks, 3, 109-118.

[42] Bishop, C.M. (1995) Probabilistic neural networks Neural Networks for Pattern Recognition. Oxford University Press

[43] Duda, R.O. and Hart, P. (1973) Pattern Classification and Scene Analysis, Wiley Interscience, New York.

[44] Bishop, C.M. (1995) Probabilistic neural networks Neural Networks for Pattern Recognition. Oxford University Press

[45] Hornik, K., Stinchcombe M. and White H. (1989)Multilayer feedforward networks are universal approximators Neural Netw., 359-366.

[46] Press, W.H., Teukolsky, S.A., Vetterling, W.T. and Flannery, B.P. (2004) Numerical Recipes in C: The Art of Scientific Computing, 2nd Ed., Cambridge University Press 
[47] Available online from: http://www.digitalglobe.com/

[48] Gillette, J.C., Stadtmiller, T.M. and Hardie, R.C. (1995)Aliasing reduction in staring infrared imagers utilizing subpixel techniques Opt. Eng., 11, 3130-3137 .

[49] Alam, M.S., Bognar, J.G., Hardie, R.C. and Yasuda, B.J. (2000)Infrared image registration and highresolution reconstruction using multiple translationally shifted aliased video frames IEEE Trans. Instr. Meas., 49, 915-923.

[50] Chiang, M.C. and Boult, T.E. (2000) Efficient super-resolution via image warping Image Vis. Comput., 18, 761-771.

[51] Gonzalez R.C. and Wintz P. (1987) Digital Image Processing, 2nd Ed., Addison-Wesley, Reading, Massachusetts

[52] Elad, M., and Hel-Or, Y., (2001) A fast super-resolution reconstruction algorithm for pure translational motion and common space-invariant blur IEEE Trans. Img. Process., 10, 1187-1193.

[53] Holst, G.C.(2007) Electro-Optical Imaging System Performance, 4th Ed., SPIE Press, JCD Publishing, Washington

[54] Park, S.C., Park, M.K. and Kang, M.G., (2003) Super-resolution image reconstruction: a technical overview IEEE Sig. Process. Mag., 20, 21-36.

[55] Vanderwalle, P., Susstrunk, S. and Vetterli,M. (2006) A frequency domain approach to registration of aliased images with applications to superresolution. EURASIP Journal on Applied Signal Processing, 2006, article ID 71459 .

[56] Gabarda, S. and Cristóbal, G. (2007) Blind image quality assessment using anisotropy J. Opt. Soc. Am. A, 24, B42-B51.

[57] Chung, J., Haber, E., and Nagy, J. (2006) Numerical methods for coupled super-resolution. Inverse Problems, 22, 1261-1272.

[58] Pham, Q.T. (2006) Spatiotonal activity in superresolution of undersampled image sequences PhD Thesis, Delft University, The Netherlands. 